Hedwig GASTEIGER, Osnabrück, Christiane BENZ, Karlsruhe

\title{
Mathematikdidaktische Kompetenz von Fachkräften im Elementarbereich - ein theoriebasiertes Kompetenzmodell
}

Die mathematikdidaktische Fachkompetenz von Fachkräften im Elementarbereich rückte in letzter Zeit zunehmend mehr in den Fokus des Forschungsinteresses. Eine Reihe von Studien beschäftigen sich mit der Struktur, dem Niveau oder der Entwicklung von professionellen Kompetenzen dieses Personenkreises im Bereich Mathematik und mit mathematikdidaktischen Überzeugungen und Praktiken. Dazu wurden bislang Modelle aus der Lehrerkompetenzforschung oder allgemeine, nicht-fachspezifische Modelle aus dem Elementarbereich adaptiert. Gerade im Hinblick auf die mathematisch-mathematikdidaktische Professionalisierung von Fachkräften im Elementarbereich oder auf die Evaluation von entsprechenden Aus- und Weiterbildungsmaßnahmen lohnt es sich, zu hinterfragen, welche Kompetenzen frühpädagogische Fachkräfte für die Gestaltung elementarerer mathematischer Bildungsprozesse wirklich brauchen und ob die bisher verwendeten Modelle professioneller Kompetenz genutzt werden können.

\section{Analyse der Anforderungen}

Um Kompetenz in einem bestimmten Bereich definieren zu können, ist es erforderlich, die Anforderungen zu analysieren, die an Personen bei der Ausübung ihrer konkreten Aufgabe gestellt werden, und dabei die entsprechenden Randbedingungen zu berücksichtigen (Blömeke et al., 2015). Für die mathematikdidaktische Kompetenz von Fachkräften im Elementarbereich heißt das zunächst, zu analysieren, welche Aspekte bei der Gestaltung früher mathematischer Bildungsprozesse maßgeblich bedeutsam sind. National herrscht hier weitgehend Konsens: Mathematische Bildung im Elementarbereich sollte bruchfreies Weiterlernen ermöglichen, trotz der Notwendigkeit zu elementarisieren fachliche Richtigkeit garantieren, sich an zentralen fundamentalen Ideen der Mathematik orientieren, über eine reine Vermittlung von Prozeduren hinausgehen und das Spiel als kindgemäße mathematische Lernaktivität berücksichtigen (Gasteiger, 2015; Kaufmann, 2010; Lorenz, 2012; Schuler, 2013; Steinweg 2008). Die zentrale Frage lautet nun, wie sich die Kompetenz des elementarpädagogischen Fachpersonals, das diese Aufgabe zu erfüllen hat, charakterisieren lässt.

\section{Kompetenz von Lehrkräften und Fachkräften im Elementarbereich}

Oelkers und Reusser (2008) verstehen unter Kompetenz „wissensbasierte Fähigkeiten in bestimmten kulturellen und lebensweltlichen Domänen", die „,in aktuelle Lern- und Problemlöseleistungen umgesetzt werden müssen“(S. In Institut für Mathematik und Informatik Heidelberg (Hrsg.), Beiträge zum Mathematikunterricht 2016 (S. x-y). Münster: WTM-Verlag 
24). Dieses Verständnis von Kompetenz lässt Dispositionen erkennen, wie Wissen oder Fähigkeiten, die darüber hinaus aber in Anwendungssituationen zur Wirkung kommen müssen (Performanz). Allerdings führt eine gewisse Disposition nicht zwangsläufig zu einer gewünschten Performanz. Es kann angenommen werden, dass zwischen Disposition und Performanz eine weitere Facette von Kompetenz erforderlich ist, die darüber entscheidet, wie erfolgreich jemand seine Fähigkeiten und sein Wissen anwenden kann. Blömeke et al. (2015) sehen sogenannte ,situation-specific skills“ in der Mittlerrolle zwischen Disposition und Performanz. Darunter fassen sie z. B. die Fähigkeit, in einer konkreten Situation wesentliche Aspekte wahrnehmen und interpretieren oder kompetente Handlungsentscheidungen treffen zu können. Modelle zur mathematikdidaktischen Kompetenz bei Lehrkräften zeigen häufig die Kompetenzstruktur von Dispositionen auf, indem verschiedene Wissensfacetten unterschieden werden (Shulman, 1987; Krauss et al., 2008), oder berücksichtigen zusätzlich die Ebene der Performanz, indem Kompetenzen zur Reflexion oder in der konkreten Handlung als Teil der Lehrerkompetenz gesehen werden (Lindmeier, 2011). Die „black box“" zwischen Disposition und Performanz bleibt bei diesen Modellen weitgehend ungeklärt. Ein gängiges Modell zur Kompetenz frühpädagogischer Fachkräfte (Fröhlich-Gildhoff et al., 2014) beschreibt mit Situationswahrnehmung und -analyse sowie mit Handlungsplanung und -bereitschaft zwei Facetten, die für die oben angesprochene „Mittlerrolle“ zwischen Disposition und Performanz relevant sein können (Situationswahrnehmung und -analyse ist im Modell jedoch als Disposition verortet). Dieses allgemeine, nicht-fachspezifische Kompetenzmodell bildet allerdings die für eine erfolgreiche Gestaltung früher mathematischer Bildungsprozesse erforderlichen Wissensfacetten nicht detailliert ab.

\section{Ein Modell zur mathematikdidaktischen Kompetenz von Fachkräf- ten im Elementarbereich}

Auf Basis der vorliegenden Kompetenzmodelle und empirischer Ergebnisse wurde ein Modell zur mathematikdidaktischen Kompetenz von Fachkräften im Elementarbereich entwickelt (s. Abb. 1, Gasteiger \& Benz, 2016). Es soll die fachlich-fachdidaktische Spezifizität abbilden und berücksichtigen, dass Bildungsprozesse im Elementarbereich in der Regel anders organisiert sind, als im Schulkontext: Lernen findet mehr in informellen Situationen statt. Dadurch sind andere Kompetenzen gefordert. Es geht weniger um ein Vorausplanen von Lerneinheiten als um ein wachsames Wahrnehmen der Aktionen und Äußerungen der Kinder, damit aus alltäglichen Situationen mathematische Lernsituationen werden (s. Abb.1, PDH). Erst in der Performanz zeigt sich die Kompetenz dieses Personenkreises in aller Deutlichkeit. Sie 
wird in Verbindung mit Fähigkeiten zur Beobachtung und Wahrnehmung in der konkreten Situation gesehen (SWB). Gerade, weil Mathematik und Mathematikdidaktik oft nicht Teil der Ausbildung der frühpädagogischen Fachkräfte ist, ist im Bereich des Wissens kritisch zu hinterfragen, welches Wissen wirklich nötig ist, um frühe mathematische Bildung gewinnbringend gestalten zu können. Es scheint durchaus vorstellbar, dass es nicht der mathematische Schulstoff ist, der hier ausschlaggebend ist, sondern u. a. ein Wissen um die mathematischen Konzepte, die im Elementarbereich von Relevanz sind, und um die entsprechenden fachlichen Linien, damit Anschlussfähigkeit des Lernens garantiert werden kann (EW). Auch zeigt sich in der Praxis, dass Fachkräfte im Elementarbereich zum Teil hervorragend mathematisches Lernen anregen, obwohl sie manchmal in eher geringem Maß über explizites Wissen verfügen. Dies lässt annehmen, dass eine Art implizites, erfahrungsbasiertes Wissen (IW) vorliegt, das den Fachkräften in Handlungssituationen ermöglicht, Performanz zu zeigen.

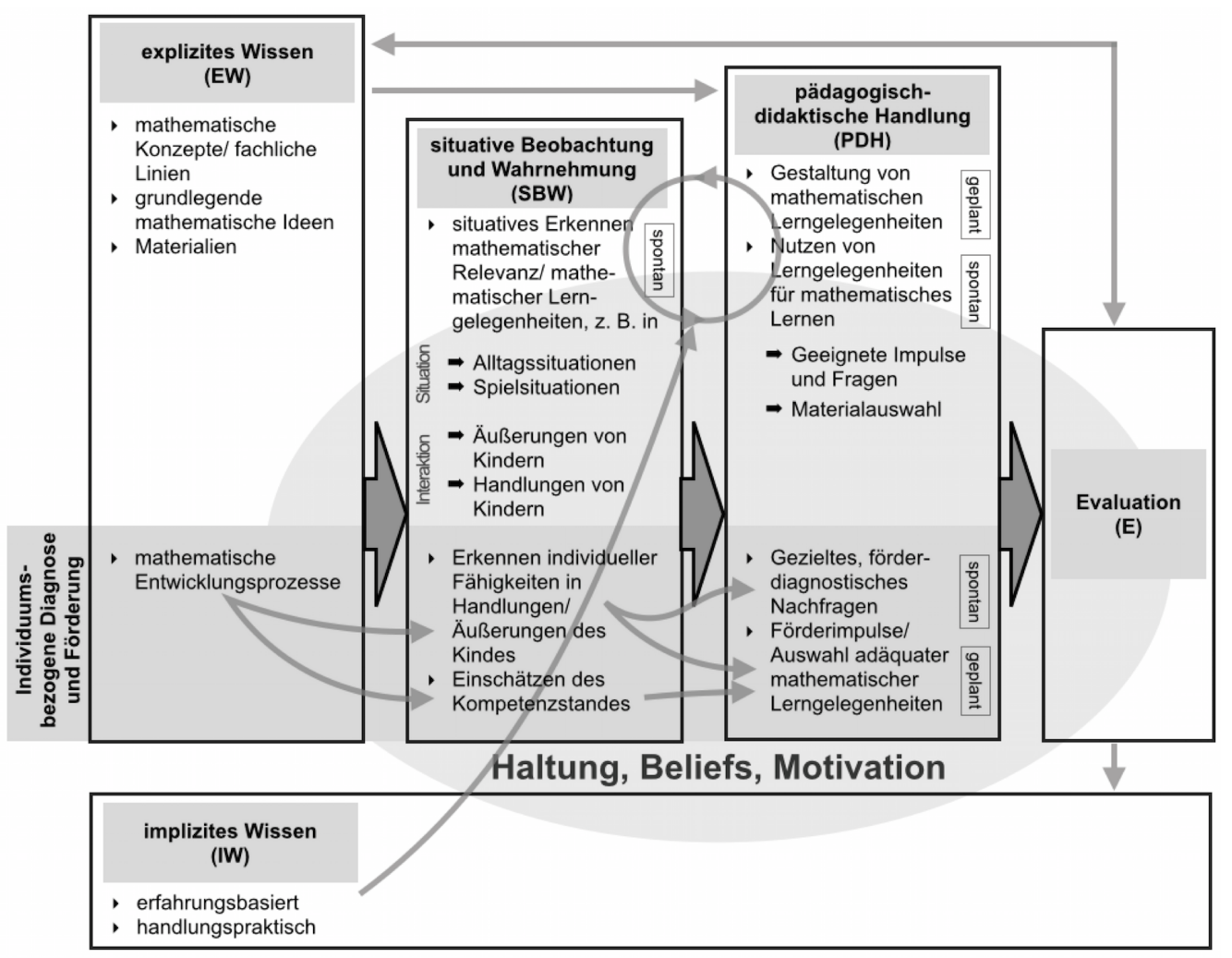

Abbildung 1: Modell zur mathematikdidaktischen Kompetenz frühpädagogischer Fachkräfte (Gasteiger \& Benz, 2016)

Im Elementarbereich kommt der kompensatorischen und der präventiven Förderung eine wichtige Rolle zu (Lorenz, 2012). Deshalb enthält das Modell die querliegende Kompetenzfacette „Individuumsbezogene Diagnose und Förderung". Sie ist wissensbasiert (EW), umfasst eine genaue Beobachtung der Kinder sowie die Wahrnehmung vorhandener Fähigkeiten (SBW) 
und zeigt sich dann in der Auswahl geeigneter mathematischer Lerngelegenheiten (PDH). Es gibt begründete Vermutungen zu Zusammenhängen zwischen den verschiedenen Kompetenzfacetten, die das Modell aufzeigt. Sie können an dieser Stelle leider nicht ausführlicher dargestellt werden - hierzu sei auf Gasteiger \& Benz (2016) verwiesen.

Perspektivisch gesehen, steht eine empirische Analyse der Kompetenzfacetten sowie der verschiedenen Zusammenhänge noch aus. Dazu ist zunächst die Analyse und Entwicklung geeigneter Erhebungsinstrumente erforderlich. Langfristig könnte das Modell - so es sich empirisch bewährt - als Grundlage für die Konzeption der Aus- und Weiterbildung der Erziehenden dienen.

\section{Literatur}

Blömeke, S., Gustafsson, J. \& Shavelson, R. J. (2015). Beyond dichotomies. Competence viewed as a continuum. Zeitschrift für Psychologie, 223(1), 3-13.

Fröhlich-Gildhoff, K., Weltzien, D., Kirstein, N., Pietsch, S., \& Rauh, K. (2014). Kompetenzen früh-/kindheitspädagogischer Fachkräfte im Spannungsfeld von normativen Vorgaben und Praxis. Freiburg: Zentrum für Kinder- und Jugendforschung.

Gasteiger, H. \& Benz, C. (2016). Mathematikdidaktische Kompetenz von Fachkräften im Elementarbereich - ein theoriebasiertes Kompetenzmodell. Journal für Mathematik-Didaktik. http://dx.doi.org/10.1007/s13138-015-0083-z

Gasteiger, H. (2015). Early mathematics in play situations: continuity of learning. In B. Perry, A. Gervasoni, \& A. MacDonald (Hrsg.), Mathematics and Transition to School. International Perspectives (S. 255-272). Singapore: Springer.

Kaufmann, S. (2010). Handbuch für die frühe mathematische Bildung. Braunschweig: Schrödel.

Krauss, S., Brunner, M., Kunter, M., Baumert, J., Blum, W., Neubrand, M., \& Jordan, A. (2008). Pedagogical content knowledge and content knowledge of secondary mathematics teachers. Journal of Educational Psychology, 100(3), 716-725.

Lindmeier, A.M. (2011). Modeling and Measuring Knowledge and Competencies of Teachers. Münster: Waxmann.

Lorenz, J. H. (2012). Kinder begreifen Mathematik. Frühe mathematische Bildung und Förderung. Stuttgart: Kohlhammer.

Oelkers, J., \& Reusser, K. (2008). Qualität entwickeln - Standards sichern - mit Differenzen umgehen. Bildungsforschung Band 27. Bonn, Berlin: BMBF.

Schuler, S. (2013). Mathematische Bildung im Kindergarten in formal offenen Situationen. Münster: Waxmann.

Shulman, L. S. (1987). Knowledge and teaching: Foundations of the new reform. Harvard Educational Review, 57(1), 1-22.

Steinweg, A. S. (2008). Zwischen Kindergarten und Schule - Mathematische Basiskompetenzen im Übergang. In F. Hellmich \& H. Köster, H. (Hrsg.), Vorschulische Bildungsprozesse in Mathematik und Naturwissenschaften (S. 143-159). Bad Heilbrunn: Klinkhardt. 